\title{
Increasing life-time of wireless sensor network using energy- efficient and fault tolerance algorithms
}

\author{
Sama Hussam Sabah, Muayad Sadik Croock
}

Computer Engineering Department, University of Technology, Baghdad, Iraq

\begin{tabular}{l} 
Article Info \\
\hline Article history: \\
Received Oct 22, 2020 \\
Revised Jul 12, 2021 \\
Accepted Jul 18, 2021 \\
\hline
\end{tabular}

\section{Keywords:}

Fault-tolerance

LEACH protocol

Sleep-scheduling mechanism

Software-engineering

approaches

WSN

\begin{abstract}
Energy-efficiency of wireless sensor networks (WSN) becomes an essential issue in the research area. This is because of the energy constraints in WSN that depend on a battery, which is difficult to replace or recharge; therefore, multiple clustering algorithms were proposed to achieve efficiency in using the available energy as much as possible. This paper proposed energyefficient and fault-tolerance algorithms that enhance the low energy adaptive clustering hierarchy (LEACH) protocol by three algorithms. The first focuses on selecting the best cluster head, and the second focuses on minimizing the required nodes within the same cluster. Simultaneously, the third fault tolerance algorithm uses software engineering techniques like sleep schedules to increase network lifetime as much as possible. The testing results of the proposed algorithms prove the claim of enhancing the lifetime of WSN. In order to check improvement of lifetime of WSN we have compered the results of the proposed algorithms with standered algorthim. The results show prove the claim of enhancing the life-time of WSN, where the total lifetime of WSN rise from about 550 rounds to reach 4100 when utilized self-checking process and rised up to 5200 after enhance minimum distans.
\end{abstract}

This is an open access article under the CC BY-SA license.

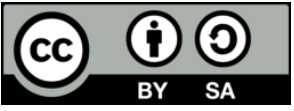

\section{Corresponding Author:}

Sama Hussam Sabah

Computer Engineering Department

University of Technology

Baghdad, Iraq

Email: 120634@student.uotechnology.edu.iq

\section{INTRODUCTION}

Lately, wireless sensor networks (WSN) is one of most active research networks areas. A WSNs are a self-organized networks comprising small nodes, depend on battery to power and they have limited range of transmission, and sink node [1], [2]. There are different applications for the sensor nodes (SNs) that can sense the physical environment for task monitoring and data collection [3]. The main disadvantage of the WSN is the limited power supply for sensor nodes, that affect directly on the network lifetime [4]. Consequently, the life-time of the WSN depends on the battery power of the nodes since each node operation consumes power [5], [6]. This means that the essential challenge in this network's design is power conservation as much as possible [7] to enhance lifetime by implementing suitable methods such as selfchecking and sleeping mode to allow more long-term work [8], [9]. To get out of the power constraint issue, the best and effective strategies discovered were clustering, which eliminated the power conservation problem [10]. Clustering strategy involves the process where the entire network is grouped into many noderelated classes. Any party named its leader as clustering head $(\mathrm{CH})$. The nodes relay data to $\mathrm{CH}$ and now $\mathrm{CH}$ is responsible for sending data to the base station (BS) in a single or multi-hop way after aggregating data 
from different nodes [11], [12]. The BS has Internet access to make data accessible to the user and to alert the public [13]. We conclude that the clustering method minimizes overhead communication, reducing power consumption and interference among sensor nodes [14], [15]. WSNs are also prone to failures, faults are unavoidable and defective sensor nodes can't do any monitoring job, so these faulty sensor nodes should be identified at the proper time and discarded from the collecting data phase to ensure the total quality of the data [16]-[18]. Hence, we focus on finding solutions these faults towards manage power consumption.

Low energy adaptive clustering hierarchy (LEACH) is the most popular routing protocol using cluster-based routing to minimize power consumption; there are also some drawbacks associated with the LEACH clustering algorithms, like selection $(\mathrm{CH})$ and cluster creation [19]. In this paper, we treat some of these drawbacks to enhance the clustering algorithm by adopting software engineering approaches and to propose a new power-efficient and fault-tolerance algorithm to prolong network lifetime and decrease power consumption in WSN. Careful steps should be taken to avoid the failure, for example, to set threshold levels of power consumption for each process to avoid any error of drain the whole available power so that the WSN must have a fault tolerance system with a high probability of fault detection and low probability of fault warning

\section{RELATED WORKS}

Decreasing power consumption in the WSN is considered a significant concern in recent decades. Accordingly, several methods have been designed to increase the life-time of the WSN and conserve available energy. Here, we focus on researches that took up two essential fields of enhancing LEACH clustering and fault-tolerance algorithms. Jassbi and Moridi [20], the authors developed a hybrid powerefficient distributed algorithm (HEED) by adding fault tolerance technique to the base station's decision on faults to take appropriate action and isolated defective nodes and take it as a value by using a weighted median process, and they can increase the corrected data rate. Salem and Shudifat [21], the authors attempted to resolve the limitations of WSN, low-cost and low-power tools that affect power exchange rates by enhancing LEACH protocol in which LEACH is extended by defining a cluster head at the shortest distance to the base station. This is to minimize power consumption in cluster head nodes and the entire network. However, more than consideration was required, such as the distance between nodes, to achieve better available power conservation. In [22], enhancement of the LEACH algorithm was proposed by not transmitting $\mathrm{CH}$ data collected from its member to a BS per round and cluster head in the next round, thereby minimizing the power efficiency and increasing network life. Compared to simple LEACH, the suggested method achieved an power-efficient gain of $200 \%$. Simple leach disadvantages, however, are still found such as never considering the gap between cluster head and base station. Arabi and Parikhani [23], the authors improved the leach protocol and considered a residual power at each node and distance between the cluster head and base station. While in [24], the researchers collected various and modern types of faults and addressed the advantages and disadvantages of each option and the acceptable solutions for each type of fault in order to achieve greater knowledge trust. Goyal and Sonal [25], the authors worked on coordinating the operation of the transceiver by using sleep scheduling to monitor power exchange effectively, using the Zigbee protocol, as it has the advantage of avoiding collisions leading to power waste.

\section{PROPOSED SYSTEM}

The disadvantages of a LEACH protocol could be identified in three issues. The first issue deals with the bad choice of cluster head. The second issue is caused by the unfair distribution sensor nodes at each cluster. Consequently, there is a wide variation in the amounts of power spent by sensor nodes in different clusters during the steady-state process. The third problem is formalized, where all sensor nodes are transmitted continuously within each cluster. Even when there is no update of sensed data, sending is performed. These three problems are the explanation for the inefficient fall of power consumption. This fall reduces the lifespan of the network. This paper proposed algorithms to reduce as much as possible the consumed power of sensor nodes and to increase network lifespan, as show in below;

\subsection{Modification of cluster head choice}

Due to important of choosing the right $\mathrm{CH}$ to manage its responsibilities, we should take care in choosing proper cluster head to achieve the best efficiency in power consumption. Since distance is the largest factor for power expenditure, we try to take the distance between $\mathrm{CH}$ and $\mathrm{BS}$ in consideration and choose the shortest distance. In addition, the residual power is considered as a factor in selecting of $\mathrm{CH}$ to increase the network life-time as shown in the flowchart in Figure 1. 


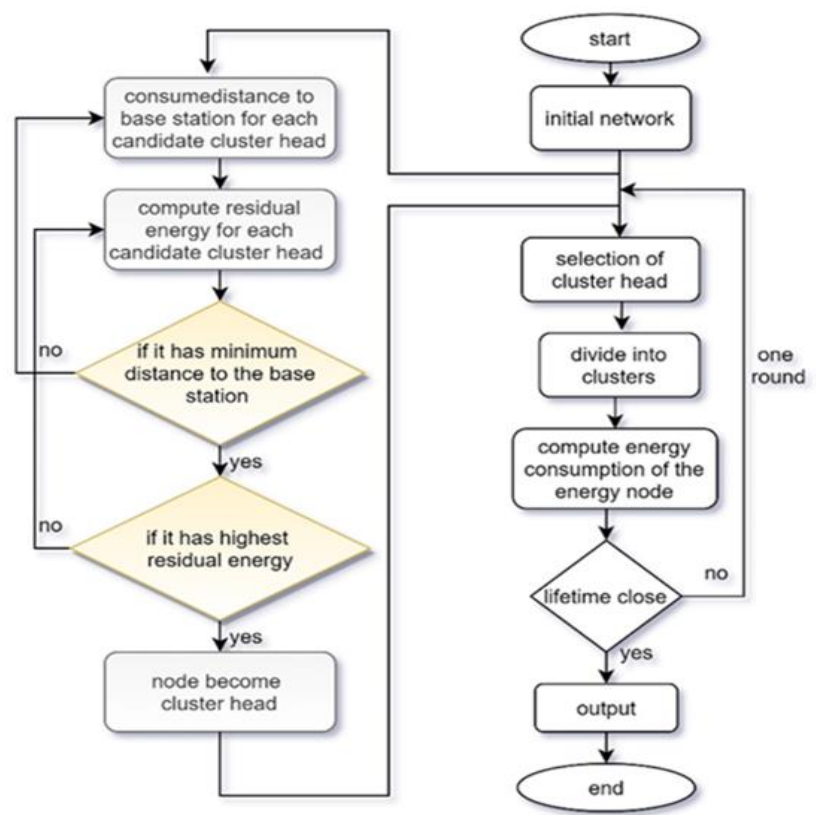

The algorithm is explained in the following step: 1.The network is initialized in the system area by random distributed of sensor nodes.

2.Implementation of the leach protocol that selects cluster heads according to the predetermined probability and number of candidates for cluster heads.

3.Choosing the node with a greater residual power and a less distant to BS.

4.Performing the leach protocol to build clusters to schedule the transmission of data between the nodes and the cluster head by using TDMA algorithm to prevent collision.

Figure 1. First leach enhancement algorithm

\subsection{Cluster fair sensor nodes distribution}

The proposed power consumption algorithm divides the WSN area into clusters with fail allocation of nodes with different types. In order to extend the lifetime of the network, the cluster approximated area is calculated. On this basis, the covered area between the members is calculated and if it is less than $20 \%$ of the total cluster area and they are from the same type, the proposed algorithm set one of them to sleep mode. Meanwhile the power of the active node is monitored, and when the power is consumed to less than threshold of $50 \%$, the active is turned to sleep mode. At the same time, the slept node is awaked to perform. This can extend the network life time to double. It is important to note that the $20 \%$ ratio is considered under the consumption of each node can cover this area instead of using two. The following flowchart in Figure 2 show the proposed algorithm steps.
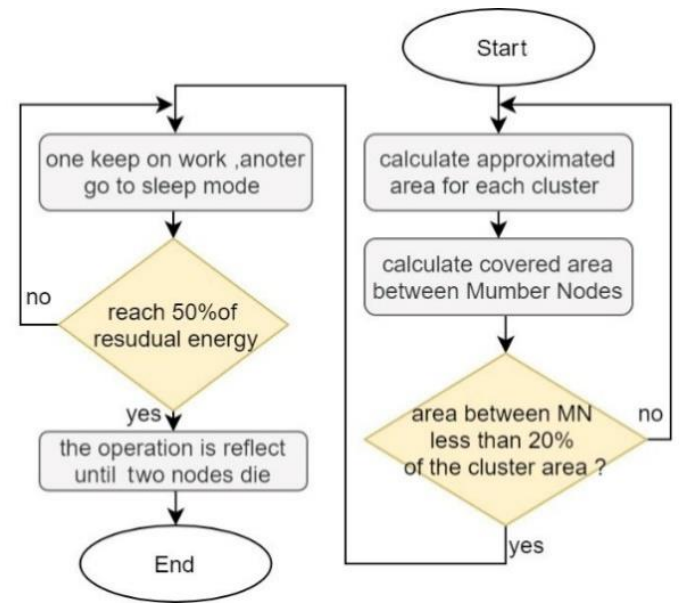

The algorithm is explained in the following steps: 1.Calculating the approximated area for each cluster.

2.Calculating covered area between member nodes (MN). In case the area is less than $20 \%$ of the cluster area, one of these Neighbor nodes is turned to sleep mode.

3.This is continued till the active node is consumed the power of $50 \%$, here the slept node is awaked and the old one is turned to sleep mode. 4.This operation is continued until two nodes are died (depleted of energy).

Figure 2. Second leach enhancement algorithm

\subsection{Fault tolerance algorithm}

As mentioned earlier, the proposed fault tolerance algorithm is used to compensate the error that can be happened in WSN. The self-checking process of software engineering in [26] is adopted for every fault in both stages (fault detection, fault recovery). This process is performed in the selected $\mathrm{CH}$. This algorithm can also increase the life-time of WSN by compensating the dead nodes, in which the WSN is still working properly. The following flowchart in Figure 3 explains the proposed fault tolerance algorithm. 
The algorithm adopted steps are explained as:

- At each sensor node, applying the self-checking method.

- Verifying that the sensor node continues to function in standard form if the residual power is greater than $70 \%$ of total power.

- Verify that sensor node manages the readings by taking a reading for each ten rounds, when the residual power of more than 30 percent and less than $70 \%$. The time period between readings makes the node to be sleep.

- Checking the residual power of the sensor node. If it is less than $30 \%$, the node is turned to sleep mode and then establish compensation process by taking the nearest neighbor nodes to calculate the average value.

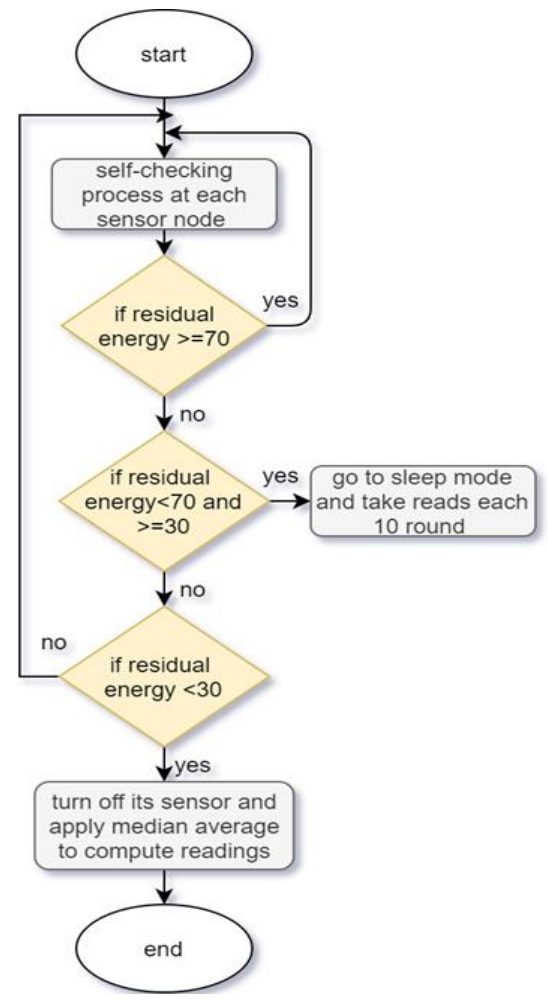

Figure 3. Proposed self-checking process algorithm

\section{RESULTS}

In order to test the proposed algorithms, we compare the result with standard LEACH protocol and other works in the same field as those in [27], [28], we created a simulator using MATLAB to verify the efficiency of them. With 100 sensor nodes allocated randomly in the region of $100 * 100$ meters, a WSN is constructed. The middle is assigned to the base station. As seen in Figure 4, each sensor node has an initial the power of 0.1 Joule, which indicates the beginning of the network operation. Each sensor node begins to join the proper cluster for data exchange at initialization. The developed and fault tolerance algorithms are continuous until all sensors are dead. The parameters used in the simulation are shown in Table 1.

We execute our proposed method with 6000 iterations to show the difference. As we note in the Figure 5, the network with basic leach protocol would drain, and all nodes die in 800 iterations; and after we added the first enhancement that takes the covered area between nodes and the residual power in the consideration, we note that the network life-time expanded to reach 1000 iterations. When we apply the proposed algorithm of cluster fair node distribution, a huge develop in network life-time to exceed 4000 iterations. At the adopting of fault tolerance in addition to previous algorithm, the WSN is still working till 5000 iterations. At the other eye, Figure 6 explains the comparison between the proposed methods and traditional LEATCH. All available power drain early, while in enhanced leach saves power to keep it alive as long as possible.and the typical distance of $\mathrm{CH}$. Nodes and BS in present case is related value depending on input. we calculate the distance ' $\mathrm{d}$ ' between the sensor node that is transmitting and the cluster head that is receiving with the following equation; 


$$
d=\operatorname{sqrt}\left((x 2-x 1)^{\wedge} 2+(y 2-y 1)^{\wedge} 2\right)
$$

where $\mathrm{x} 2$ and $\mathrm{y} 2$ the coordinates the cluster head and $\mathrm{x} 1$ and $\mathrm{y} 1$ the coordinates of the transmitting node.

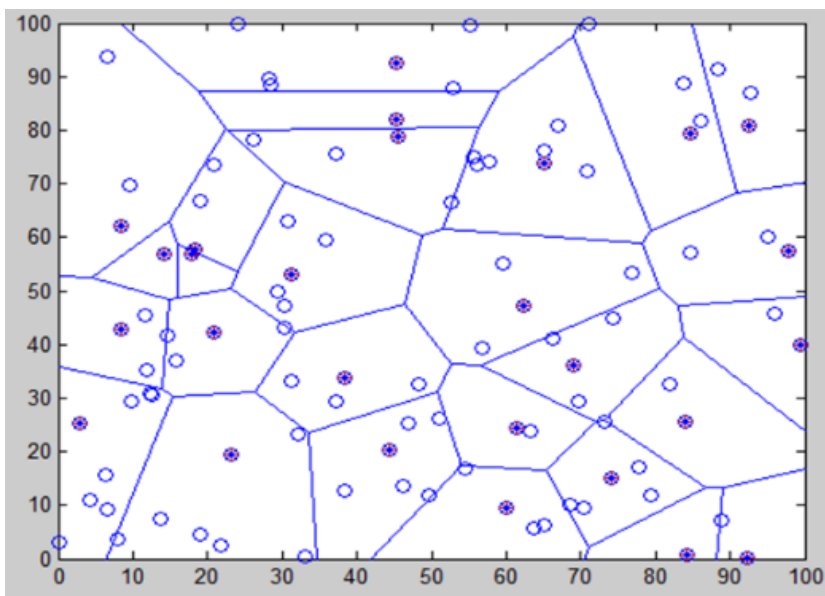

\begin{tabular}{|cc|}
\hline$*$ & Base Station \\
Sensor Node \\
& Cluster head \\
& Cluster border
\end{tabular}

Figure 4. The beginning of the network

Table 1. Simulation parameters used in the system

\begin{tabular}{lc}
\hline \multicolumn{1}{c}{ Parameters } & Values \\
\hline Area's length & $100 \mathrm{~m}$ \\
Area's width & $100 \mathrm{~m}$ \\
x coordination of base station & Length / 2 \\
y coordination of base station & Width / 2 \\
Number of sensors in the area & 100 \\
Rounds number & 6000 \\
Initial energy of each node & $0.1 \mathrm{~J}$ \\
Power for transferring each bit & $50 * 0.000000001$ \\
Powwr for receiving of each bit & $50 * 0.000000001$ \\
Power for Data aggregation & $5 * 0.000000001$ \\
\hline
\end{tabular}

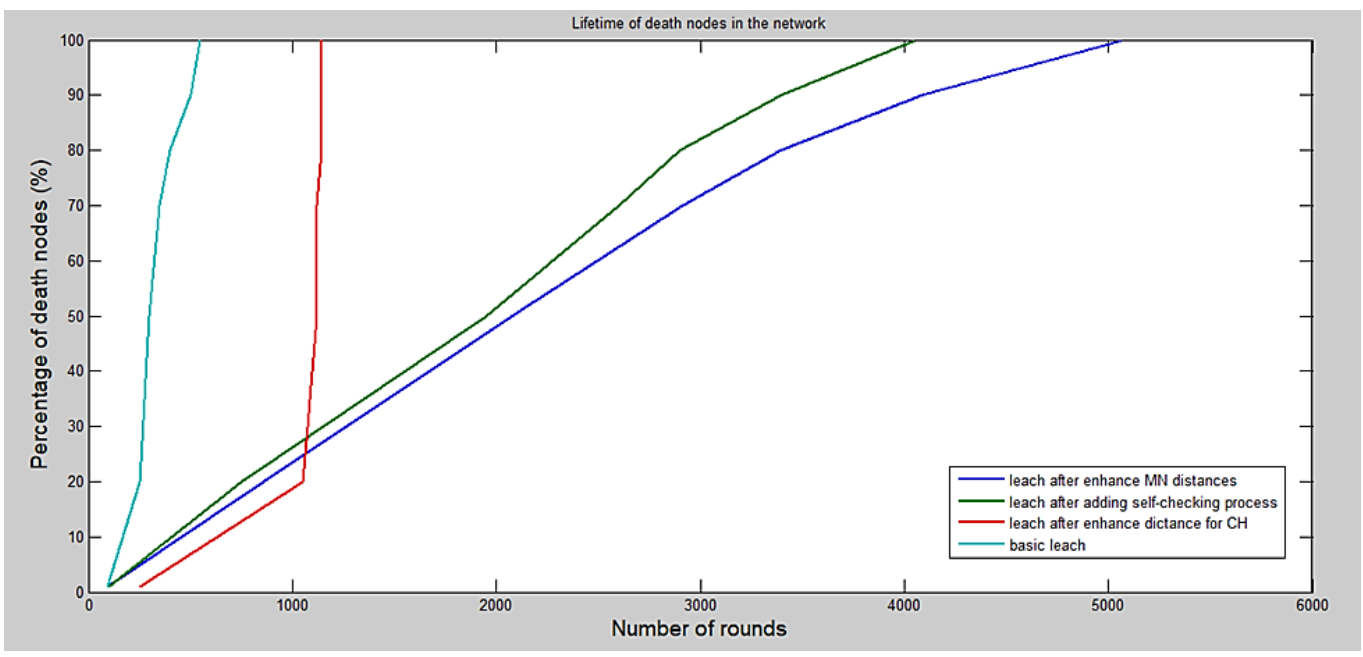

Figure 5. Composition of the lifetime improvement of the nodes between basic leach and proposed enhanced algorithms. The compartion shows the dead of nodes (its battery has run out) with rounds 


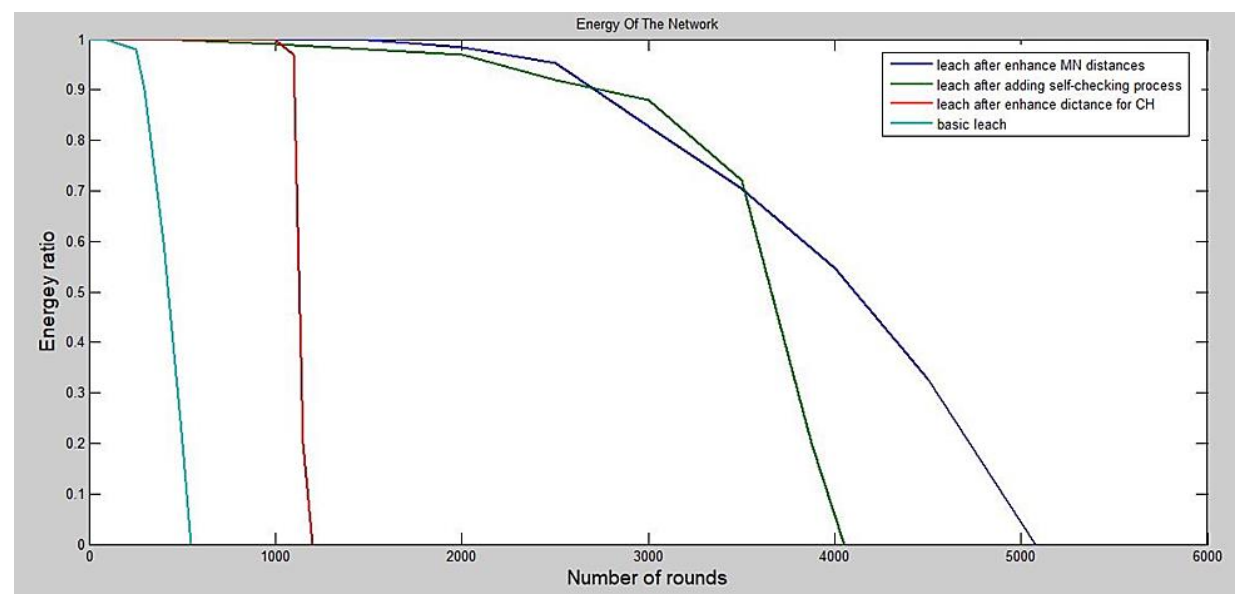

Figure 6. Composition of basic leach and proposed enhanced algorithms in term of power rates

\section{CONCLUSION}

In this paper, three algorithms have been proposed for improving the routing procedures in the WSN LEACH protocol. The first solution was to pick the correct cluster head node at each round for each cluster. This was done by modifying the LEACH algorithm by taking the distance between candidate $\mathrm{CH}$ and BS also the residual power in the consideration when select cluster head. Also, the overheads of cluster head are minimized by minimized the number of generated clusters and the variance of the number of cluster members. The clusterhead change Algorithm is designed to minimize changes. Cluster heads only change when two clusterheads come into contact, or when a node becomes disconnected from all clusterheads. The second approach was designed to solve the problem of distribution of unequal sensor nodes to monitor the different amounts of power exchanges in clusters. It also managed the closed nodes from the same type to be turned to sleep or active mode to increase the WSN life-time. The third approach used fault tolerance approach to controlling the work of nodes inside the clusters depending on the residual power. The achieved results explained the development in performance in terms of WSN life-time after applying the proposed algorithms. The results are considered acceptable according to the proposed study compared with basic LEACH protocol. MATLAB software gave scope to test it. In the future, it is possible to introduce security concepts to the wireless sensor network in order to make it more reliability and show the impact on its performance and calculate power consumption rates.

\section{REFERENCES}

[1] E. Abedini and Rezai.Abdalhossein, "A modified digital to digital encoding method to improve the Wireless Body Area Network (WBAN) transmission," 2015 2nd International Conference on KBEI, 2015, doi: 10.1109/KBEI.2015.7436193.

[2] M. S. Talib, "Minimizing the energy consumption in wireless sensor networks," Journal of University of Babylon for Pure and Applied Sciences, vol. 26, no. 1, pp. 17-28, 2018, doi: 10.29196/jub.v26i1.349.

[3] H. Mohapatra and A. K. Rath, "Fault tolerance in WSN through PE-LEACH protocol," IET Wirel. Sens. Syst., vol. 9, no. 6, pp. 358-365, 2019, doi: 10.1049/iet-wss.2018.5229.

[4] G. Pradeebaa and N. Lavanis, "Network lifetime improvement using routing algorithm with sleep mode in wireless sensor network,” Proc. 2016 IEEE Int. Conf., WiSPNET, 2016, pp. 1572-1575, doi: 10.1109/WiSPNET.2016.7566401.

[5] S. Anand and S. Sinha, "A customised approach for reducing energy consumption in wireless sensor network," International Journal of Innovative Technology and Exploring Engineering, vol. 8, no. 8, pp. 1427-1431, 2019.

[6] A. F. Marhoon and M. A. Zubaidie, "Reduce Energy Consumption by Improving the LEACH Protocol," International Journal of Computer Science and Mobile Computing, vol. 3, no. 1, pp. 1-09, 2014.

[7] K. P. Ferentinos, T. A. Tsiligiridis, and K. G. Arvanitis, "Energy optimization of wireless sensor networks for environmental measurements," Proc. 2005 IEEE Int. Conf. Comput. Intell. Meas. Syst. Appl. CIMSA 2005, vol. 2005, no. July, 2005, pp. 250-255, doi: 10.1109/CIMSA.2005.1522872.

[8] R. Wan, N. Xiong, and N. T. Loc, "An energy-efficient sleep scheduling mechanism with similarity measure for wireless sensor networks," Human-centric Comput. Inf. Sci., vol. 8, no. 1, 2018, doi: 10.1186/s13673-018-0141-x.

[9] D. S. Park, "Fault tolerance and energy consumption scheme of a wireless sensor network," Int. J. Distrib. Sens. Networks, vol. 2013, no. March, 2013, doi: 10.1155/2013/396850.

[10] P. C. Srinivasa Rao and H. Banka, "Energy efficient clustering algorithms for wireless sensor networks: novel chemical reaction optimization approach," Wireless Networks, vol. 23, no. 2, pp. 433-452, 2017, doi: 10.1007/s11276-015-1156-0.

[11] S. Devi, I. Ahmed, and M. Urvashi, "Optimization Technique to Improve the Energy Efficiency in WSN : LEACHMGWO,” IJARIIE, no. 5, pp. 176-182, 2018. 
[12] M. Elshrkawey, S. M. Elsherif, and M. Elsayed Wahed, "An Enhancement Approach for Reducing the Energy Consumption in Wireless Sensor Networks," J. King Saud Univ. - Comput. Inf. Sci., vol. 30, no. 2, pp. 259-267, 2018, doi: 10.1016/j.jksuci.2017.04.002.

[13] V. Sharma, P. Rajpoot, A. Gupta, K. Dubey, N. Pandey, and N. Verma, "Heterogeneous clustering for energy optimization in wireless sensor networks," Proc. 9th Int. Conf. Cloud Comput. Data Sci. Eng. Conflu. 2019, 2019, pp. 92-99, doi: 10.1109/CONFLUENCE.2019.8776933.

[14] T. Rault, "Energy-efficiency in wireless sensor networks," Thèse présentée pour 1 ' obtention du grade de Docteur de 1 ' UniversitédeTechnologie de Compiègne, 2016.

[15] Z. Beiranvand, A. Patooghy, and M. Fazeli, "I-LEACH: An efficient routing algorithm to improve performance \& to reduce energy consumption in wireless sensor networks," IKT 2013 5th Conf. Inf. Knowl. Technol., 2013, pp. 13-18, doi: 10.1109/IKT.2013.6620030.

[16] S. H. Sabah and M. S. Croock, "Increasing WSN Lifetime using Clustering and Fault Tolerance Methods," Iraqi Journal for Electrical and Electronic Engineering, vol. 17, no. 1, 2021, pp. 94-99, doi: 10.37917/ijeee.17.1.11.

[17] Z. Zhang, A. Mehmood, L. Shu, Z. Huo, Y. Zhang, and M. Mukherjee, "A survey on fault diagnosis in wireless sensor networks," IEEE Access, vol. 6, pp. 11349-11364, 2018, doi: 10.1109/ACCESS.2018.2794519.

[18] M. Dong, H. Li, Y. Li, Y. Deng, and R. Yin, "Fault-tolerant topology with lifetime optimization for underwater wireless sensor networks," Sadhana, vol. 45, no. 1, 2020, doi: 10.1007/s12046-020-01406-1.

[19] M. Elshrkawey, S. M. Elsherif, M. E. Wahed, "An Enhancement Approach for Reducing the Energy Consumption in Wireless Sensor Networks," Journal of King Saud University - Computer and Information Sciences, vol. 30, no. 2, pp. 259-267, 2018, doi 10.1016/j.jksuci.2017.

[20] S. J. Jassbi and E. Moridi, "Fault Tolerance and Energy Efficient Clustering Algorithm in Wireless Sensor Networks: FTEC,” Wirel. Pers. Commun., vol. 107, no. 1, pp. 373-391, 2019, doi: 10.1007/s11277-019-06281-6.

[21] A. O. A. Salem and N. Shudifat, "Enhanced LEACH protocol for increasing a lifetime of WSNs," Personal and Ubiquitous Computing, vol. 23, no. 5-6, pp. 901-907, 2019, doi: 10.1007/s00779-019-01205-4.

[22] S. A. Mohammed, K. E. Aly, and A. M. Ghuniem, "An enhancement process for reducing energy consumption in wireless sensor network," Int. J. Emer. Tren. Eng. Res., vol. 8, no. 6, pp. 2765-2769, 2020, doi: 10.30534/ijeter/2020/89862020.

[23] Z. Arabi and R. Parikhani, "EFP : New Energy-Efficient Fault- Tolerant Protocol for Wireless Sensor Network," Int. J. of Comp. Nets. and Comm., vol. 4, no. 6, pp. 111-119, 2012, doi: 10.5121/ijenc.2012.4609.

[24] A. Choudhary, S. Choudhary, and A. Mishra, "Review on Fault Tolerance in Wireless Sensor Network," International Journal of Computer Applications, vol. 182, no. 45, pp. 22-25, 2019, doi: 10.5120/ijca2019918597.

[25] D. Goyal and Sonal, "Power management in Wireless Sensor Network," 2016 3rd International Conference on Computing for Sustainable Global Development (INDIACom), 2016.

[26] M. S. Croock, S. D. Khuder, and Z. A. Hassan, "Self-checking method for fault tolerance solution in wireless sensor network," International Journal of Electrical and Computer Engineering, vol. 10, no. 4, pp. 4416-4425, 2020, doi: 10.11591/ijece.v10i4.pp4416-4425.

[27] X. Qu, "Energy Efficient Wireless Sensor Network with Modified LEACH Algorithm," Master Thesis, Department of Electrical Engineering, Blekinge Institute of Technology, Karlshamn, Swedia, 2012.

[28] T. M. Behera, S. K. Mohapatra, U. C. Samal, M. S. Khan, M. Daneshmand, and A. H. Gandomi, "Residual energybased cluster-head selection in WSNs for IoT application," IEEE Internet Things J., vol. 6, no. 3, pp. 5132-5139, 2019, doi: 10.1109/JIOT.2019.2897119.

\section{BIOGRAPHIES OF AUTHORS}

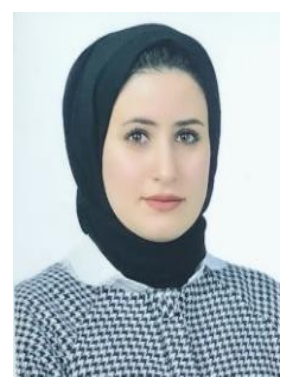

Sama Hussam Sabah Received B.sc from the university of technology in 2017. I Have work experience with software development for three years to develop and maintain websites as a full-stack developer, build a website with high specifications, and have good experience in computer network and pc hardware maintenance; I got an IFC certificate in Effective communication and writing a good cv. also have a certified to Participate in in the Power of Computer Science initiative I look forward to gaining more experience. A student in the research stage to obtain a master's degree in the computer engineering department.

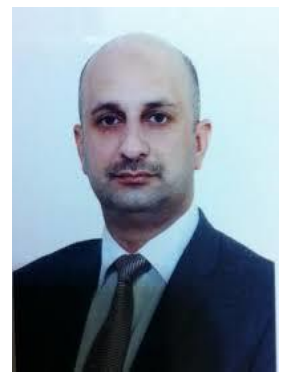

Muayad Sadik Croock. He is an assistant professor of computer engineering/ software engineering at university of technology. He received his B.Sc. from university of technology, Iraq at 1998 and 2003, respectively. He got his PhD from Newcastle University, UK at 2012. His research interestings include WSN, Software Engineering, Security and cyber-security. 